\title{
Importance of glutamate dehydrogenase stimulation for glucose and glutamine synthesis in rabbit renal tubules incubated with various amino acids ${ }^{\circ}$
}

\author{
Katarzyna Winiarska, Przemysław Bożko, Tadeusz Lietz and Jadwiga Bryła ${ }^{\circledR}$ \\ Institute of Biochemistry, Warsaw University, Warszawa, Poland
}

Received: 02 July, 1998

Key words: rabbit kidney-cortex tubules, glutamate dehydrogenase, $\mathrm{BCH}$, amino acids, gluconeogenesis, glutamine synthesis

\begin{abstract}
The effect of 2-aminobicyclo[2.2.1]heptan-2-carboxylic acid (BCH), an L-leucine nonmetabolizable analogue and an allosteric activator of glutamate dehydrogenase, on glucose and glutamine synthesis was studied in rabbit renal tubules incubated with alanine, aspartate or proline in the presence of glycerol and octanoate, i.e. under conditions of efficient glucose formation. With alanine+glycerol+octanoate the addition of $\mathrm{BCH}$ resulted in a stimulation of alanine and glycerol consumption, accompanied by an increased glucose, lactate and glutamine synthesis. In contrast, when alanine was substituted by either aspartate or proline, $\mathrm{BCH}$ altered neither glucose formation nor glutamine and glutamate synthesis, while an accelerated glycerol utilization was accompanied by a small increase in lactate production. In view of the BCH-induced changes in intracellular metabolite levels the acceleration of gluconeogenesis by $\mathrm{BCH}$ in the presence of alanine+glycerol+octanoate is probably due to (i) increased uptake of alanine via alanine aminotransferase, (ii) stimulation of phosphoenolpyruvate carboxykinase, a key-enzyme of gluconeogenesis, (iii) rise of glucose-6-phosphatase activity, as well as (iv) activation of the malate-aspartate shuttle resulting in an augmented glycerol utilization for lactate and glucose synthesis.
\end{abstract}

In rabbit and guinea pig kidneys amino acids such as L-alanine, L-aspartate, L-glutamate and L-proline are not utilized for gluconeogenesis when applied as the sole substrates,

\footnotetext{
This work was supported partially by the Polish State Committee for Scientific Research (Grant No. 6 P04A 024 08).

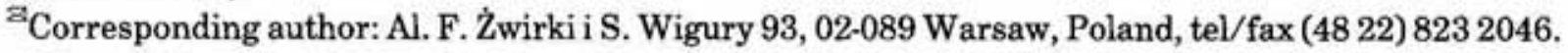

Abbreviations: $\mathrm{BCH}, 2$-aminobicyclo[2.2.1]heptan-2-carboxylic acid; DABS-Cl, 4-dimethylaminoazobenzene-4'-sulfonyl chloride; DHAP, dihydroxyacetone phosphate; MSO, L-methionine sulfoximine.
} 
while glutamine synthesis is very efficient [1-5]. In the previous reports from this laboratory it has been shown that in rabbit renal tubules incubated with alanine, aspartate, glutamate or proline glucose is produced when glycerol or lactate and fatty acids or ketone bodies are also provided [3-5]. The addition of the latter substrates stimulates gluconeogenesis by a mechanism involving a decrease in glutamate dehydrogenase activity followed by a decline of glutamine formation.

Therefore, the aim of this investigation was to study both glucose and glutamine synthesis in the presence of 2-aminobicyclo[2.2.1]heptan-2-carboxylic acid (BCH), a nonmetabolizable Lleucine analogue, reported as an activator of glutamate dehydrogenase for both the isolated enzyme [6] and various cells: hepatocytes [7], $\beta$-pancreatic cells [8, 9] and brain synaptosomes [10].

\section{MATERIAL AND METHODS}

\section{Isolation and incubation of kidney-} cortex tubules. Fed rabbits (male, California strain, 2-3 kg body weight) were anaesthetized with pentobarbital ( $30 \mathrm{mg}$ per $\mathrm{kg}$ body weight). Renal cortical tubules were isolated as described previously [11] and incubated (about $10-15 \mathrm{mg}$ dry weight) at $37^{\circ} \mathrm{C}$ in $2 \mathrm{ml}$ of Krebs-Ringer buffer in $25 \mathrm{ml}$ plastic Erlenmeyer flasks sealed with rubber stoppers under the atmosphere of $95 \% \mathrm{O}_{2}+5 \% \mathrm{CO}_{2}$. Substrates were added at concentrations given in legends to Tables. Octanoate was applied as an emulsion in a bovine serum albumine solution. The reaction was stopped by the addition of $35 \%$ perchloric acid ( 0.1 vol. of tubule suspension).

Analytical methods. Samples for metabolite determination were withdrawn after 60 min of incubation. The intracellular content of metabolites was estimated in samples following the centrifugation of tubule suspension through silicone oil into perchloric acid solution as described previously [11]. Glucose pro- duction was measured spectrophotometrically with glucose oxidase and peroxidase [12]. Amino acids were determined by HPLC after derivatization with DABS-Cl [13]. Metabolite levels were measured spectrophotometrically or fluorimetrically by standard enzymatic techniques [12]. Isotopic studies of the incorporation of various substrates into glucose were performed according to Exton \& Park [14] and Pilkis et al. [15].

Enzymes and chemicals. Collagenase (typ IV) was purchased from Sigma Chemical Co. (St. Louis, U.S.A.). Enzymes for metabolite determinations were obtained from Boehringer (Mannheim, Germany) or Sigma Chemical Co. [U- ${ }^{14} \mathrm{C}$ ]alanine, [U- $\left.{ }^{14} \mathrm{C}\right]$ aspartate and $\left[\mathrm{U}-{ }^{14} \mathrm{C}\right]$ glycerol were from Amersham Searle Corporation (Amersham, Great Britain). Amino acids, MSO and DABS-Cl were purchased from Sigma Chemical Co., while BCH was from Calbiochem (La Jolla, U.S.A.). Other reagents were from P.O.Ch. (Gliwice, Poland).

\section{RESULTS AND DISCUSSION}

\section{Glucose, glutamine, glutamate and lactate synthesis}

As in rabbit renal tubules amino acids such as alanine, proline or aspartate are efficiently utilized for glucose formation in the presence of glycerol and either fatty acids [5] or ketone bodies [4], rabbit kidney-cortex tubules were incubated with various amino acids, glycerol and octanoate. Both glucose and glutamate synthesis from alanine+glycerol+octanoate or proline+glycerol+octanoate were about twice lower than from aspartate+glycerol+octanoate, while glutamine accumulation was similar (Table 1), but lower than in the presence of amino acids alone due to a decline of glutamate dehydrogenase activity resulting in a diminished formation of $\mathrm{NH}_{4}{ }^{+}$ions for glutamine synthesis [3-5]. In order to activate this enzyme $2 \mathrm{mM} \mathrm{BCH}$ was applied. At this concentration the compound stimulated ammo- 
nia production by about $30 \%$ (not shown) in the presence of MSO, an inhibitor of glutamine synthetase [16].

As shown in Table 1, in renal tubules incubated with aspartate+glycerol+octanoate or proline+glycerol+octanoate the addition of 2 $\mathrm{mM} \mathrm{BCH}$ did not alter the rate of glucose, glutamine and glutamate synthesis and only slightly accelerated lactate formation. In contrast, when aspartate or proline was substituted by alanine, $2 \mathrm{mM}$ BCH resulted in an increase of both glucose and glutamine synthesis by about $25 \%$ and $40 \%$, respectively, while lactate production was only slightly stimulated and glutamate formation unaltered. Moreover, in renal tubules incubated with alanine+glycerol+octanoate $\mathrm{BCH}$ augmented the incorporation of $\left[\mathrm{U}^{14} \mathrm{C}\right]$ alanine and [U${ }^{14} \mathrm{Clglycerol}$ into glucose by $17 \%$ and $26 \%$, respectively (Table 2). In contrast, when alanine was replaced by aspartate, the utilization of both glycerol and aspartate for glucose synthesis did not change following the addition of $\mathrm{BCH}$.

$\mathrm{BCH}$-induced stimulation of glucose production in spite of an accelerated glutamine formation is opposed to our previous findings indicating that the activation of gluconeogenesis is caused by a diminished utilization of amino-acid carbon skeletons for glutamine production in the presence of glycerol or lactate and fatty acids or ketone bodies [3-5].
Thus, the stimulatory effect of $\mathrm{BCH}$ on the rate of glucose formation is due to a mechanism which does not involve a decrease in glutamine synthesis.

\section{Substrate utilization}

As presented in Table 3, in the absence of $\mathrm{BCH}$ the utilization of alanine and aspartate was similar. The addition of $\mathrm{BCH}$ did not change the rate of aspartate removal from the incubation medium while it augmented the utilization of alanine by about $40 \%$. In view of this observation, an increase in alanine utilization might contribute to the stimulatory effect of $\mathrm{BCH}$ on glucose and glutamine synthesis in renal tubules incubated with alanine+glycerol+octanoate (cf. Table 1). The addition of $\mathrm{BCH}$ caused also a slight increase in glycerol utilization (by about $10-20 \%$ ) in renal tubules incubated with alanine+glycerol+octanoate contributing to the acceleration of lactate formation and glucose synthesis (cf. Table 1). A slight acceleration of glycerol uptake in the presence of aspartate+glycerol+octanoate was also accompanied by a stimulation of lactate production (cf. Table 1).

\section{Intracellular metabolite levels}

To identify the mechanisms responsible for the stimulatory effect of $\mathrm{BCH}$ on gluconeo-

Table 1. The effect of BCH on glucose, glutamine, glutamate and lactate synthesis in renal tubules incubated with various amino acids in the presence of glycerol and octanoate

\begin{tabular}{lccccc}
\hline \multirow{2}{*}{ Amino acid } & \multirow{2}{*}{$\mathrm{BCH}$} & Glucose & Glutamine & Glutamate & Lactate \\
\cline { 3 - 7 } & & \multicolumn{5}{c}{$(\mu \mathrm{mol} / \mathrm{h}$ per g dry wt. $)$} \\
\hline \multirow{2}{*}{ Alanine } & + & $56.0 \pm 7.1$ & $71.1 \pm 6.0$ & $43.7 \pm 9.0$ & $67.0 \pm 6.4$ \\
& + & $73.1 \pm 10.7^{\mathrm{a}}$ & $99.7 \pm 6.2^{\mathrm{a}}$ & $39.9 \pm 8.3$ & $77.8 \pm 7.6$ \\
Aspartate & + & $109.2 \pm 23.7$ & $83.9 \pm 13.4$ & $83.6 \pm 12.3$ & $48.7 \pm 3.2$ \\
& - & $116.6 \pm 23.7$ & $95.1 \pm 19.2$ & $95.2 \pm 12.4$ & $58.9 \pm 3.8^{\mathrm{a}}$ \\
Proline & + & $48.2 \pm 12.0$ & $66.8 \pm 3.0$ & $53.1 \pm 4.1$ & $84.8 \pm 3.7$ \\
& + & $54.6 \pm 14.6$ & $71.2 \pm 4.9$ & $60.8 \pm 6.8$ & $102.2 \pm 5.9^{\mathrm{a}}$ \\
\hline
\end{tabular}

Renal tubules were incubated for $60 \mathrm{~min}$. Glycerol and BCH were added at $2 \mathrm{mM}$ concentrations, while amino acids and octanoate at $1 \mathrm{mM}$ and $0.5 \mathrm{mM}$, respectively. Values are means \pm S.D. for $3-10$ experiments. ${ }^{\mathrm{n}} P<0.05 v \mathrm{~s}$. corresponding controls with no BCH. 
Table 2. The effect of $\mathrm{BCH}$ on the incorporation of ${ }^{14} \mathrm{C}$-labeled substrates into glucose in renal tubules incubated with glycerol, octanoate and either alanine or aspartate

\begin{tabular}{|c|c|c|c|}
\hline${ }^{14} \mathrm{C}$-Labeled substrate & Unlabeled substrate & $\mathrm{BCH}$ & $\begin{array}{c}{\left[^{14} \mathrm{C}\right] \text { Glucose synthesis }} \\
\text { ( } \mu \mathrm{mol}^{14} \mathrm{C}_{3} \text { units/h per } \mathrm{g} \text { dry wt.) }\end{array}$ \\
\hline \multirow{2}{*}[\mathrm{U}^{14}\mathrm{C}]{$\mathrm{Ala}$} & \multirow{2}{*}{ Glycerol } & - & $17.8 \pm 1.6$ \\
\hline & & + & $21.0 \pm 1.1^{\mathrm{a}}$ \\
\hline \multirow{2}{*}[\mathrm{U}^{14}\mathrm{C}]{ Asp } & \multirow{2}{*}{ Glycerol } & - & $31.7 \pm 6.7$ \\
\hline & & + & $39.1 \pm 2.8$ \\
\hline \multirow{2}{*}[\mathrm{U}^{14}\mathrm{C}]{$\mathrm{Glycerol}$} & \multirow{2}{*}{ Ala } & - & $101.2 \pm 13.6$ \\
\hline & & + & $127.9 \pm 13.6^{\mathrm{a}}$ \\
\hline \multirow{2}{*}[\mathrm{U}^{14}\mathrm{C}]{ Glycerol } & \multirow{2}{*}{ Asp } & - & $155.1 \pm 17.1$ \\
\hline & & + & $153.1 \pm 16.7$ \\
\hline
\end{tabular}

Experimental conditions were as described in the legend to Table 1 . Values are means \pm S.D. for 3 experiments. ${ }^{a} P<0.05$ vs. corresponding controls with no $\mathrm{BCH}$.

genesis and glutamine formation from alanine+glycerol+octanoate we measured the intracellular metabolite levels both in the absence and presence of $\mathrm{BCH}$.

Among intracellular metabolites one of the most marked changes in concentration was observed for 2-oxoglutarate in spite of the lack of changes in glutamate concentration (Table 4). $\mathrm{BCH}$ diminished the intracellular level of 2 oxoglutarate by about $30 \%$ and $20 \%$ in the presence of alanine+glycerol+octanoate and aspartate+glycerol+octanoate, respectively. Since with pyruvate as substrate 2-oxoglutarate level did not change on addition of $\mathrm{BCH}$ (not shown), the $\mathrm{BCH}$-induced decrease in the intracellular content of this metabolite in the presence of alanine+glycerol+octanoate might be related to an acceleration of alanine utiliza- tion (cf. Table 3) via alanine aminotransferase utilizing 2-oxoglutarate as substrate. In the presence of alanine+glycerol+octanoate a decline of 2-oxoglutarate concentration was accompanied by about 2-fold decrease in malate concentration and about 2 -fold increase in phosphoenolpyruvate level. Therefore, it seems likely that the $\mathrm{BCH}$-induced acceleration of glucose synthesis under these conditions might be due to the activation of phosphoenolpyruvate carboxykinase activity, a key-enzyme of gluconeogenesis, resulting from a decrease of intracellular 2-oxoglutarate, an inhibitor of the enzyme [17]. This hypothesis is also supported by diminished intracellular levels of malate (by about $40 \%$ ) and citrate (by about $25 \%$ ), suggesting more efficient utilization of oxaloacetate by phosphoe-

Table 3. The effect of $\mathrm{BCH}$ on glycerol and amino acids utilization in renal tubules incubated with glycerol and octanoate in the presence of alanine or aspartate

\begin{tabular}{|c|c|c|c|}
\hline \multirow{2}{*}{ Amino acid } & \multirow{2}{*}{$\mathrm{BCH}$} & Glycerol & Amino acid \\
\hline & & \multicolumn{2}{|c|}{$(\mu \mathrm{mol} / \mathrm{h}$ per $\mathrm{g}$ dry wt. $)$} \\
\hline \multirow{2}{*}{ Alanine } & - & $106.9 \pm 12.1$ & $155.6 \pm 12.4$ \\
\hline & + & $121.8 \pm 8.8^{\mathrm{a}}$ & $213.2 \pm 5.5^{\mathrm{a}}$ \\
\hline \multirow{2}{*}{ Aspartate } & - & $149.6 \pm 5.6$ & $166.9 \pm 18.0$ \\
\hline & + & $162.0 \pm 2.5^{\mathrm{a}}$ & $169.9 \pm 16.0$ \\
\hline
\end{tabular}

Experimental conditions were as described in the legend to Table 1. Values are means \pm S.D. for $3-5$ experiments. ${ }^{4} P<0.05$ vs. corresponding controls with no $\mathrm{BCH}$. 
nolpyruvate carboxykinase in comparison with that by malate dehydrogenase and citrate synthase. In contrast, in renal tubules incubated with aspartate+glycerol+octanoate intracellular levels of phosphoenolpyruvate and citrate did not change following the addition of $\mathrm{BCH}$, while malate content was only slightly decreased (by about 15\%). Since with aspartate+glycerol+octanoate the intracellular concentration of 2-oxoglutarate in the absence of $\mathrm{BCH}$ was by about $20 \%$ lower than with alanine+glycerol+octanoate, the activity of phosphoenolpyruvate carboxykinase was probably not impaired under the former conditions.

Since 2-oxoglutarate is also an efficient competitive inhibitor of aspartate formation via aspartate aminotransferase [18], a diminished concentration of 2-oxoglutarate might result in a stimulation of flux of cytosolic reducing equivalents into mitochondria via the malate-aspartate shuttle. This is in agreement with an increased level of aspartate inside renal tubules incubated with alanine+glycerol+octanoate (from $2.1 \pm 0.1$ to $3.1 \pm 0.1$, without and with $\mathrm{BCH}$, respectively, $P<0.05$ for 5 experiments). Moreover, as the efficient transport of NADH through the inner mitochondrial membrane is essential for glycerol utilization as a glucose precursor [19], it seems likely that the activation of the malateaspartate shuttle leading to a fall of cytosolic $\mathrm{NADH} / \mathrm{NAD}^{+}$ratio and an enhancement of glycerol-3-phosphate dehydrogenase activity might contribute to the stimulatory effect of $\mathrm{BCH}$ on glucose formation from glycerol in the presence of alanine+glycerol+octanoate (cf. Table 2). In agreement with this suggestion, the addition of $\mathrm{BCH}$ to renal tubules incubated with alanine+glycerol+octanoate caused a decrease in [glycerol-3-phosphate]/[DHAP] ratio (from 767.6 \pm 75.4 to $604.3 \pm 53.2$, without and with $\mathrm{BCH}$, respectively, $P<0.05$ for 5 experiments), while [lactate]/[pyruvate] ratio was unaffected $(12.7 \pm 0.4$ and $11.8 \pm 1.0$, without and with $\mathrm{BCH}$, respectively, $P<0.05$ for 5 experiments). These observations confirm previous findings indicating that $\mathrm{NADH} / \mathrm{NAD}^{+}$pools utilized by lactate and glycerol-3-phosphate dehydrogenases are poorly connected $[3,4$, 20].

In the presence of $\mathrm{BCH}$ we also observed a diminished concentration of glucose-6-

Table 4. The effect of $\mathrm{BCH}$ on the intracellular levels of metabolites in renal tubules incubated with glycerol and octanoate in the presence of alanine or aspartate

\begin{tabular}{lccc}
\hline \multirow{2}{*}{ Metabolite } & BCH & Alanine & Aspartate \\
\cline { 3 - 4 } 2-Oxoglutarate & - & $1.50 \pm 0.08$ & $(\mu$ mol per g dry wt. $)$ \\
& + & $1.03 \pm 0.08^{\mathrm{a}}$ & $1.21 \pm 0.12$ \\
Glutamate & - & $14.03 \pm 2.68$ & $0.89 \pm 0.04^{\mathrm{a}}$ \\
& + & $14.05 \pm 2.48$ & $26.25 \pm 0.95$ \\
PEP & - & $0.15 \pm 0.02$ & $23.80 \pm 1.20$ \\
& + & $0.27 \pm 0.03^{\mathrm{a}}$ & $0.38 \pm 0.04$ \\
Malate & - & $0.52 \pm 0.09$ & $0.43 \pm 0.02$ \\
& + & $0.30 \pm 0.07^{\mathrm{a}}$ & $0.48 \pm 0.05$ \\
Citrate & - & $1.41 \pm 0.10$ & $0.39 \pm 0.04^{\mathrm{a}}$ \\
& + & $1.06 \pm 0.03^{\mathrm{a}}$ & $0.88 \pm 0.04$ \\
\hline
\end{tabular}

Experimental conditions were as shown in the legend to Table 1. Renal tubules were separated from the reaction medium as described in Material and Methods. Values are means \pm S.D. for $3-4$ experiments. " $P<0.05$ vs. corresponding controls with no BCH. 
phosphate inside renal tubules incubated with alanine+glycerol+octanoate $(0.50 \pm 0.03$ and $0.37 \pm 0.02$, without and with $\mathrm{BCH}$, respectively, $P<0.05$ for 5 experiments) accompanied by an increased glucose content $(3.49 \pm 0.15$ and $4.08 \pm 0.20$, without and with $\mathrm{BCH}$, respectively, $P<0.05$ for 5 experiments). Since 2-oxoglutarate in the presence of $\mathrm{Mg}^{2+}$ ions has been reported as an inhibitor of glucose-6-phosphatase [21, 22] it is possible that the $\mathrm{BCH}$-induced decrease in the intracellular content of this metabolite might also result in an augmented glucose-6-phosphatase activity contributing to the acceleration of glucose production.

In summary, the results presented in this paper indicate that the flux through glutamate dehydrogenase may limit both glucose formation and glutamine production in the presence of alanine+glycerol+octanoate, in contrast to aspartate+glycerol+octanoate and proline+ glycerol +octanoate. Therefore, stimulation of this enzyme activity on the addition of an Lleucine analogue results in an increase of both glucose and glutamine synthesis in rabbit renal tubules incubated with alanine+glycerol+octanoate.

The authors are very grateful to Dr. Jan Zaleski for the gift of $\mathrm{BCH}$ and to Miss Bożena Dąbrowska for excellent technical assistance.

\section{REFERENCES}

1. Michoudet, C., Martin, G. \& Baverel, G. (1988) Pyruvate carboxylation in glutamine synthesis from alanine by isolated guinea-pig renal cortical tubules. Pflugers Arch. 412, 7-11.

2. Baverel, G., Martin, G. \& Michoudet, C. (1990) Glutamine synthesis from aspartate in guineapig renal cortex. Biochem. J. 268, 437-442.

3. Lietz, T. \& Bryła, J. (1995) Glycerol and lactate induce reciprocal changes in glucose formation and glutamine production in isolated rabbit kidney-cortex tubules incubated with as- partate. Arch. Biochem. Biophys. 321, 501509 .

4. Lietz, T., Winiarska, K. \& Bryła, J. (1997) Ketone bodies activate gluconeogenesis in isolated rabbit renal cortical tubules incubated in the presence of amino acids and giycerol. Acta Biochim. Polon. 44, 323-332.

5. Lietz, T., Rybka, J. \& Bryla, J. (1998) Fatty acids and glycerol are required to induce gluconeogenesis from alanine in isolated rabbit renal cortical tubules. Amino Acids (in press).

6. Fahien, L.A., Teller, J.K., Macdonald, M.J. \& Fahien, C.M. (1990) Regulation of glutamate dehydrogenase activity by $\mathrm{Mg}^{2+}$ and magnification of leucine activation by $\mathrm{Mg}^{2+}$. Mol . Pharmacol. 37, 943-949.

7. Zaleski, J., Wilson, D.F. \& Erecinska, M. (1986) Glutamine metabolism in rat hepatocytes. Stimulation by nonmetabolizable ana$\log$ of leucine. J. Biol. Chem. 261, 1408214090.

8. Sener, A. \& Malaisse, W.J. (1980) L-Leucine and nonmetabolized analogue activate pancreatic islet glutamate dehydrogenase. Nature 288, 187-189.

9. Sener, A., Leclercq-Meyer, V., Giroix, M.H., Malaisse, W.J. \& Hellerstrom, C. (1987) Opposite effects of D-glucose and nonmetabolized analogue of Lleucine on respiration and secretion in insulin-producing tumoral cells (RINm5F). Diabetes 36, 187-192.

10. Erecinska, M. \& Nelson, D. (1990) Activation of glutamate dehydrogenase by leucine and its nonmetabolizable analogue in rat brain synaptosomes. J. Neurochem. 54, 1335-1343.

11. Zabłocki, K., Gemel, J. \& Bryła, J. (1983) The inhibitory effect of octanoate, palmitate and oleate on glucose formation in rabbit kidney tubules. Biochim. Biophys. Acta 757, 111-118.

12. Bergmeyer, H.U. (1965) Methods of Enzymatic Analysis. Verlag Chemie, Weinheim, Academic Press, New York, London. 
13. Chang, J., Kneht, R. \& Braun, D.G. (1983) Amino acid analysis in picomole range by precolumn derivatization and high-performance liquid chromatography. Methods Enzymol. 91, 41-48.

14. Exton, J.H. \& Park, C.R. (1967) Control of gluconeogenesis in liver. J. Biol. Chem. 242, 2622-2636.

15. Pilkis, S.J., Riou, J.P. \& Claus, T.H. (1976) Hormonal control of $\left[{ }^{14} \mathrm{C}\right]$ glucose synthesis from $\left[{ }^{14} \mathrm{C}\right]$ dihydroxyacetone and glycerol in isolated liver hepatocytes. J. Biol. Chem. 251, 7841-7852.

16. Meister, A. (1984) Enzymology of glutamine; in Glutamine Metabolism in Mammalian Tissues (Häussinger, D. \& Siess, H., eds.) pp. 315, Springer-Verlag, Berlin, Heidelberg.

17. Titheradge, M.A., Picking, R.A. \& Haynes, R.C., Jr. (1992) Physiological concentrations of 2-oxoglutarate regulate the activity of phosphoenolpyruvate carboxykinase in liver. Biochem. J. 285, 767-771.

18. Strzelecki, T., Strzelecka, D., Koch, C.D. \& LaNoue, K.F. (1988) Sites of action of glucagon and other $\mathrm{Ca}^{2+}$ mobilizing hormones on the malate-aspartate cycle. Arch. Biochem. Biophys. 264, 310-320.

19. Berry, M.N., Kun, E. \& Werner, H.V. (1973) Regulatory role of reducingequivalent transfer from substrate to oxygen in the hepatic metabolism of glycerol and sorbitol. Eur. J. Biochem. 33, 407-417.

20. Berry, M.N., Philips, J.W. \& Grivell, A.R. (1992) Interactions between mitochondria and cytoplasm in isolated hepatocytes. Curr. Top. Cell. Reg. 33, 309-328.

21. Mithieux, G., Vega, F.V. \& Riou, J.-P. (1990) The liver glucose-6-phosphatase of intact microsomes is inhikited and displays sigmoid kinetics in the presence of $\beta$-ketoglutaratemagnesium and oxaloacetate-magnesium chelates. J. Biol. Chem. 265, 20364-20368.

22. Minassian, C., Ajzannay, A., Riou, J.-P. \& Mithieux, G. (1994) Investigation of the mechanism of glycogen rebound in the liver of 72-hour fasted rats. J. Biol. Chem. 269, 16585-16588. 